\title{
Advances in diagnostic interventional pulmonology
}

Nassar Al-Zubaidi, Ayman O. Soubani

Department of Internal Medicine, Division of Pulmonary, Critical Care and Sleep Medicine, Wayne State University School of Medicine, Detroit, Michigan, USA

\begin{tabular}{|c|}
\hline Access this article online \\
\hline Website: www.avicennajmed.com \\
\hline DOI: 10.4103/2231-0770.160229 \\
\hline Quick Response Code: \\
\hline
\end{tabular}

\section{ABSTRACT}

The recent advances in diagnostic pulmonary procedures have revolutionized the evaluation of abnormal thoracic findings including lung nodules and masses, mediastinal lymphadenopathy, and pleural diseases. Bronchoscopies with endobronchial ultrasonography and electromagnetic navigation are examples of new technology that has significantly improved the specificity and sensitivity of these procedures in diagnosis and staging of lung cancer without the need for more invasive procedures. This report describes the different diagnostic pulmonary interventions providing a description of the procedures, their indications, diagnostic yield and drawback.

Key words: Bronchoscopy, diagnosis, pulmonary nodules

\section{INTRODUCTION}

Diagnostic procedures utilized by the pulmonologist have undergone major improvements in the last several years, and today, a wide variety of those is available and frequently developed [Table 1]. Bronchoscopy has been in use for decades but has been remodeled many times, to improve its diagnostic yield. Nowadays, the pulmonologist can access mediastinal lymph nodes with the help of a new technology; endobronchial ultrasound (EBUS). We can now reach peripheral lung lesions using different techniques, including radial probe (RP) EBUS, navigational bronchoscopy or virtual bronchoscopy. The pleural disease can be investigated with the use of medical pleuroscopy (MP).

Standard bronchoscopic techniques consist of rigid bronchoscopy and flexible bronchoscopy. Gustav Killian is credited with the introduction of the rigid bronchoscope in 1898 when he used it to extract a pork bone from the right main bronchial stem of a patient. ${ }^{[1,2]}$ Once the flexible bronchoscope (FB) became available, the diagnostic uses of rigid bronchoscope became limited, and it is now used for therapeutic purposes.

\section{FLEXIBLE BRONCHOSCOPY WITH OR WITHOUT FLUOROSCOPY}

\section{Description}

First described by Dr. Shigeto Ikeda in 1966, the FB has been the corner stone of most of the pulmonary diagnostic procedures. Since then, the FB has undergone major improvements. A miniature video camera at the proximal end of the FB was added in 1987. In most centers, the FB is attached to a separate screen, which allows the physician to have a better image quality and maneuverability. Fluoroscopy has been routinely used as an adjunct to the FB when obtaining transbronchial lung biopsies of peripheral lesions. ${ }^{[3,4]}$

\section{Indications and yield}

The FB has been used for a wide array of clinical indications: Sampling endobronchial lesions for diagnosis of cancer or sarcoidosis, obtaining bronchoalveolar lavage samples for diagnosis of infectious causes or malignancy, performing transbronchial needle aspiration (TBNA) of lymph nodes, or from lung masses or nodules or transbronchial lung biopsies to diagnose interstitial lung disease are some of the major indications. ${ }^{[5]}$

The yield of bronchoalveolar lavage for the diagnosis of pneumonia varies between $30 \%$ and $75 \%$, but when it comes to mycobacterial infections, tuberculous or non-tuberculous, the yield is around $70 \%$ on average. ${ }^{[6]}$ Transbronchial lung biopsies increase the yield of bronchoalveolar lavage in diagnosing infectious causes in the immunocompromised patients, to around 70\%. ${ }^{[7,8]}$ Transbronchial lung biopsies also an overall diagnostic utility of around $70 \%$ for diffuse

Address for correspondence: Dr. Ayman O. Soubani, Division of Pulmonary, Critical Care and Sleep Medicine, Wayne State University School of Medicine, Harper University Hospital, 3990 John R - 3 Hudson, Detroit, MI 48201, USA. E-mail: asoubani@med.wayne.edu 


\begin{tabular}{l} 
Table I: Currently available diagnostic interventional \\
pulmonary procedures \\
\hline Diagnostic pulmonary procedures \\
\hline Flexible bronchoscopy \\
RP-EBUS \\
CP-EBUS \\
ENB \\
Virtual bronchoscopy \\
NBI \\
Autofluorescence bronchoscopy \\
MP \\
\hline RP-EBUS=Radial probe endobronchial ultrasound, CP-EBUS=Curvilinear probe \\
endobronchial ultrasound, ENB=Electromagnetic navigational bronchoscopy, \\
NBI=Narrow band imaging, MP=Medical pleuroscopy
\end{tabular}

lung disease. ${ }^{[9]}$ The yield has been shown to be better with the use of fluoroscopy when trying to sample lung masses. ${ }^{[10]}$

Transbronchial needle aspiration also has a wide range of diagnostic yield, reported in most studies to be anywhere between $40 \%$ and $80 \%$ for detecting malignant mediastinal disease. ${ }^{[11]}$

\section{Drawbacks and complications}

Flexible bronchoscopy is a relatively safe procedure. Complications such as pneumothorax, bleeding, hypoxia, or cardiopulmonary arrest happen in $<1 \%$. Patient selection should be carefully made, with attention to the patient's respiratory status, the presence of hypoxia, cardiac disease or diffuse lung disease such as severe emphysema. ${ }^{[4]}$ In certain situations, the patient might need to be intubated for safer procedure handling if the procedure is deemed necessary. One should also pay attention to the presence of bleeding diathesis and medication profile, especially in the case of anticoagulants and antiplatelet such as clopidogrel. ${ }^{[12]}$

Flexible bronchoscopy remains a limited technique when it comes to sampling mediastinal lesions, lymph nodes, and peripheral lung lesions. Fluoroscopy does aid in guiding the needle, but it does not provide three-dimensional images for accurate sampling of lesions. Furthermore, pneumothorax rate does not seem to be lower with the use of fluoroscopy. ${ }^{[10]}$

\section{Endobronchial ultrasound}

Two types of EBUS have been recently developed. The first EBUS was a radial probe (RP) EBUS. Eventually, a curvilinear probe (CP) EBUS was introduced and has been more frequently used.

\section{RADIAL PROBE ENDOBRONCHIAL ULTRASOUND}

\section{Description}

Use of ultrasound with Bronchoscopy started with the use of RP ultrasound, first described in 1992. ${ }^{[13]}$ The RP is a small $1.4 \mathrm{~mm}$ probe that is fitted into the working channel of a $\mathrm{FB}$, permitting a $360^{\circ}$ visualization of the surrounding structures. Available frequencies are $20 \mathrm{MHz}$ and $30 \mathrm{MHz}$. The most widely used $20 \mathrm{MHz}$ probe has a penetration depth of up to $5 \mathrm{~cm}$, and needs a $2.8 \mathrm{~mm}$ working channel, but a peripheral type bronchoscope can fit a smaller, ultra miniature probe, into a $2.0 \mathrm{~mm}$ working channel, allowing the scope to be inserted further into the bronchial tree, for evaluation of peripheral lesions.

A major barrier to obtaining good image quality was the presence of air between the probe and the bronchial wall. This is overcome by supplying the probe tip with a water-filled balloon sheath.

Another advancement was the introduction of guide sheath in 2003 to aid with peripheral type EBUS. This larger sheath contains the probe structures, leaving only the probe ultrasound to be free. Once the lesion location is confirmed by the ultrasound, the probe is then pulled while the guide sheath stays in place. The working channel through which the probe was inserted is then used for biopsy forceps and brushes.

\section{Indications and yield}

Radial probe ultrasound (RP-EBUS) has been used for the following indications: Assessment of the involvement of bronchial wall in tumor invasion, transbronchial biopsy of mediastinal lymph nodes and/or mediastinal lesions, and evaluation and biopsy of peripheral lung nodules.

The use of RP-EBUS was first described in the evaluation of layers of central airways. Kurimoto et al. examined the normal tissue of 45 specimens of the bronchial wall to determine correlation with EBUS findings. He then compared the EBUS findings of 24 cases of lung cancer involving the airways, with the histopathological findings, to determine the accuracy of the depth diagnosis on EBUS. A good correlation between histology and EBUS characterization of the normal bronchial structure was found. Also, depth diagnosis was the same in $95 \%$ of the 24 cases. ${ }^{[14]}$ Similar findings were reported by Tanaka et al., who found a 93\% diagnostic accuracy among 15 studied patients. ${ }^{[15]}$ Baba et al. was also able to identify tumor invasion easily as hypoechoic areas, especially if the cartilage layer is used as a reference to evaluate the rest of the bronchial wall. ${ }^{[16]}$

Herth et al. concluded in their study, which compared computed tomography (CT) assessment of thoracic tumor invasion into bronchial wall versus RP-EBUS assessment of the same in 131 patients, that RP-EBUS has far better 
specificity (100\%) sensitivity (89\%) and accuracy (94\%) compared to CT scan (28\%, $75 \%$, and $51 \%$ respectively). The ability of chest CT and EBUS to distinguish between compression and infiltration was measured against the histology results. ${ }^{[17]}$

The determination of tumor invasiveness into bronchial or tracheal wall helps making management decisions and staging early lung cancers. Lesions that do not cross the cartilaginous layer can be treated for example with photodynamic therapy, whereas those that go beyond that layer may require surgery or radiation therapy.

With the advent of CP real-time EBUS, RP EBUS is rarely used these days in TBNA of mediastinal nodes or masses. However, it remains a well-validated tool for such procedure.

Herth et al. are accredited with many of the earlier studies that evaluated the validity of RP-EBUS for that use. From 1999 to 2000, they studied the use of this technique in 242 patients with mediastinal or hilar lymphadenopathy, successfully obtaining samples in $86 \%$, independent of lymph node size or location. Diagnostic accuracy was $72 \%$ of those sampled. ${ }^{[18]}$ In a later randomized study, Herth et al. compared this technique to bronchoscopic blind techniques, and concluded that EBUS significantly increases the yield of TBNA in all stations except in the sub carinal region (EBUS had 86\% yield compared with $74 \%$ for the conventional method in the sub carinal region, and $84 \%$ compared to $58 \%$ in other stations). The number of necessary needle passes was also lower. ${ }^{[19]}$ A cross-over study comparing RP EBUS with transesophageal ultrasound in 160 patients with mediastinal lymphadenopathy, resulted in similar diagnostic yields, although the transbronchial approach was superior for right-sided lymph nodes. Combining both approaches provides results similar to those of mediastinoscopy. ${ }^{[20]}$

The most recent use of RP-EBUS is for the purpose of sampling small peripheral lung nodules, not reachable otherwise by conventional bronchoscopy with fluoroscopy. As described earlier, a smaller bronchoscope with a $2.0 \mathrm{~mm}$ working channel carries the EBUS probe to smaller bronchi. The normal air-filled lung tissue surrounding the bronchi will appear in the shape of white homogenous lines and circles around the probe, known as the "snow storm" appearance. A lesion or nodule will disrupt that architecture and will appear mostly hypo echoic with hyper echoic lines between the lesion and normal tissue. ${ }^{[21,22]}$

In one study, the use of RP EBUS for small peripheral lesions was evaluated in comparison to fluoroscopy. A total of
138 patients, 54 patients had small lesions that were not seen by fluoroscopy, and for which the diameter was an average of $2.2 \mathrm{~cm}$. Of those, $89 \%$ were visualized by RP-EBUS and in $70 \%$, a biopsy established the diagnosis. The only complication was pneumothorax in one patient. This shows the efficacy of RP-EBUS in the diagnosis of small peripheral lesions not reachable by fluoroscopy. ${ }^{[23]}$

These findings were re-demonstrated by Kurimoto et al. in his study of 150 patients with small peripheral lesions. EBUS yielded similar results (average of 70\%) with or without successful fluoroscopy. ${ }^{[24]}$ In that same study, the efficacy of using a guide sheath was demonstrated as well. The yield was reported to be $77 \%$ after studying all 150 patients. The diagnostic yield, however, was significantly higher when the probe was within the lesion (87\%), than when it was adjacent to it (42\%). ${ }^{[24]}$

Since inter-study variability in terms of yield is well recognized, one systematic review and meta-analysis of 16 studies involving 1420 patient with peripheral lesions, and studying the yield of RP-EBUS, was done. EBUS had a point specificity of 1.0 and pointed sensitivity of 0.73 for the detection of cancer. Prevalence of malignancy, lesion size, and reference standard used were the main factors responsible for inter-study heterogeneity for sensitivity. ${ }^{[25]}$

The question whether EBUS is comparable to CT guided biopsy remains to be answered. Most studies, including a meta-analysis of 3052 lesions from 39 studies, still show evidence that transthoracic CT guided biopsy had a better diagnostic accuracy. ${ }^{[27]}$ However, the rate of complications is much lower with RP-EBUS when compared to CT guided biopsy. ${ }^{[26]}$

The RP EBUS has also been mentioned in the literature for other indications. Evaluating airway thickness in the setting of Asthma follow-up might help identifying patients with advanced severe Asthma. The use of EBUS in relapsing polychondritis was also reported. In this case, cartilaginous damage can be identified on EBUS image. ${ }^{[27]}$

\section{CURVILINEAR PROBE REAL TIME ENDOBRONCHIAL ULTRASOUND}

\section{Description}

The CP EBUS is a curved probe that can be flexed to certain angles. The probe that is built as part of the bronchoscope is attached to the distal end of the device and its fibers occupy a $2.0 \mathrm{~mm}$ working channel of a scope that has $6.8 \mathrm{~mm}$ outer diameter. It is usually directed at a $30-40^{\circ}$ angle, allowing a view in $90^{\circ}$ angle. A water-filled balloon is usually used 
to help stabilizing the probe against the bronchial wall and providing a better image quality. The ultrasound usually has the capability to run Doppler mode, allowing better characterization of visualized structures. After identifying the target node or lesion, the needle is then pushed forward until hooked inside the lesion [Figure 1]. One could see the biopsy needle as it enters the lesion and moves back and forth to obtain samples, a real-time work mode that this EBUS type has, as an advantage over RP-EBUS.

\section{Indications and yield}

The main use of CP-EBUS is for sampling mediastinal lesions (masses and lymph nodes) and central peri-bronchial lesions. This primarily aids in the diagnosis and staging of lung cancer. Other helpful diagnostic uses include the diagnosis of sarcoidosis and chronic infections.

Since its first use in 2004 by Yasufuko, CP-EBUS has been gaining increasing popularity amongst pulmonologists. He was able to report sensitivity and specificity of 95 and $97 \%$ in distinguishing benign from malignant causes of mediastinal and hilar lymphadenopathy. ${ }^{[28]}$

Many studies that followed confirmed similar results. $\mathrm{Gu}$ et al., in their meta-analysis of 11 studies involving 1299 patients, reported a pooled sensitivity of $93 \%$ and specificity of $100 \%$ when used for the purpose of staging lung cancer ${ }^{[29]}$ Dong et al. also reported pooled sensitivity and specificity of 90 and $99 \%$ respectively, in their meta-analysis of 9 studies involving 1099 patients diagnosed with nonsmall cell lung cancer. ${ }^{[30,31]}$

A study comparing EBUS guided TBNA with Mediastinoscopy enrolled 153 patients and found excellent agreement between both techniques in $91 \%$ of patients and similar sensitivity

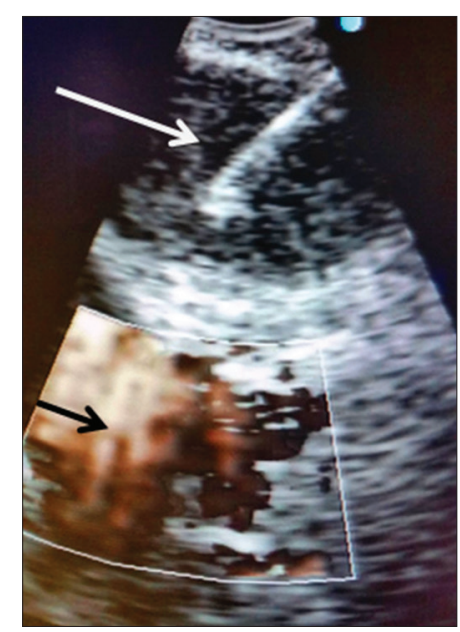

Figure 1: The image shows needle inserted in subcrinal lymph node under ultrasound guidance (white arrow) and vascular structure detected by Doppler (black arrow) and specificity. More complications were observed in the mediastinoscopy arm. ${ }^{[32]}$ This was also observed in other smaller studies that found similar results. ${ }^{[33]}$

When trying to identify patients with inoperable cancers, EBUS TBNA had similar yield when compared to positron emission tomography (PET) or CT scan of the chest. A study of 79 patients with the potentially operable disease, whether suspected or proven lung cancer, compared the use of all three modalities in distinguishing those from inoperable disease. Each test was interpreted blindly. The sensitivity of CT was $43 \%$, but PET and EBUS TBNA had similar sensitivities of 68 and $63 \%$, respectively. However, EBUS TBNA proved to be more specific than PET (100\% vs. $70 \%$ ) with considerably lower cost. ${ }^{[34]}$

There were some doubts about EBUS TBNA being useful for the diagnosis of sarcoidosis since this technique uses a smaller needle in comparison to the regularly blinded technique. However, one study compared the use of EBUS TBNA versus blinded regular Bronchoscopy in 50 patient randomized to either procedure. The results were in favor of EBUS with a sensitivity and specificity of $83 \%$ and $100 \%$, respectively as compared to $61 \%$ and $100 \%$ to that of regular bronchoscopy. ${ }^{[35]}$

In a meta-analysis of 15 studies involving 533 patients, CP EBUS had a diagnostic yield of $54-93 \%$ with a pooled accuracy of around 79\%. ${ }^{[36]}$

\section{Drawbacks and complications of endobronchial ultrasound}

Complications of EBUS are much similar to those of regular flexible bronchoscopy. In Japan, where the use of EBUS is widespread, one article studying 455 facilities, reported an overall complication rate of $1.23 \%$ in the case of EBUS guided TBNA, with hemorrhage being the most frequent. If anything, pneumothorax rate remains much lower than that of CT-guided transthoracic needle aspiration (TTNA). This was demonstrated in a meta-analysis of 16 studies of RP-EBUS involving 1420 patients, where pneumothorax rate was also at $1 \%$, as compared to a rate as high as $15 \%$ to that of CT-guided needle aspiration. ${ }^{[37]}$ EBUS, on the other hand, is a procedure that is commonly performed under general anesthesia, which is associated with its own potential complications.

Radial probe EBUS has the limitation of lacking real-time imaging as discussed above. Also, it can be difficult, for the probe to locate small peripheral pulmonary lesions, particularly in the upper lobes, because of the scope angulation required. ${ }^{[28,38]}$ 
Training and cost effectiveness are also issues that are considered. The procedure requires the presence of highly trained personnel and support staff, as well as a physician who has in-depth experience. ${ }^{[28]}$

\section{ELECTROMAGNETIC NAVIGATIONAL BRONCHOSCOPY}

\section{Description}

This technique was developed for the $1^{\text {st }}$ time by Schwarz et al. who first described it in an animal model in $2003 .{ }^{[39]}$ Chest CT scan images are first loaded into a computer program that constructs a three-dimensional model. This is called the planning phase. Next, the patient's chest is placed between three magnetic fields. The bronchoscope helps advancing a locatable guide, which is usually is handled by a knob located proximally and can turn it into different directions. The locatable guide is pushed through a bronchial tract already constructed in the planning phase. The magnetic field helps maneuvering the guide and correlating its position to the tract, by providing information about its location on a virtual three-dimensional image, and this is also displayed on a screen which has sagittal, coronal, and axial views [Figure 2]. Once the guide is close to the lesion, the locatable guide is removed, and sampling instruments (brush, needle or forceps) are inserted through the guide sheet that is left in place. $^{[40]}$

\section{Indic ations and yield}

The main indication for the navigational bronchoscopy is obtaining samples from peripheral lesions otherwise not reachable by regular bronchoscopy. The same group that did the animal study in 2003 carried out the first human study in 2006. They tested the procedure on 13 patient and were able to obtain positive biopsy results in 9 of the

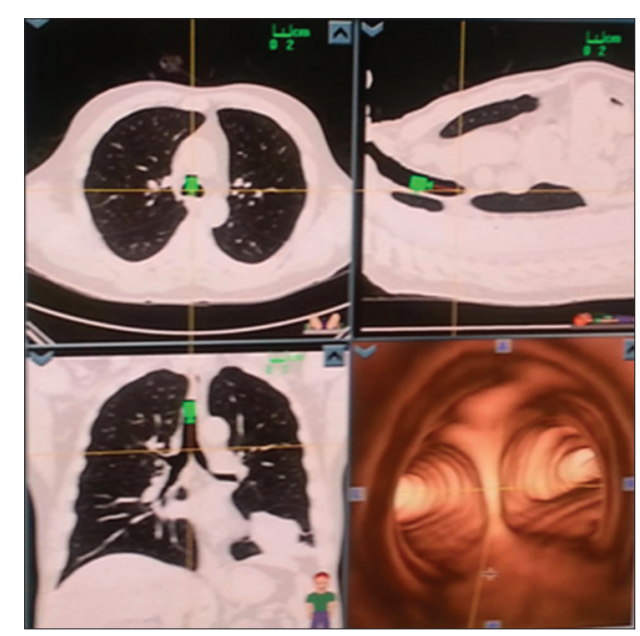

Figure 2: Electromagnatic navigational bronchosocpy preplanning screen with four viewports showing axial, coronal and sagittal computed tomography views and virtual bronchoscopy view
13 cases. ${ }^{[41]}$ Several small studies have been published since then, where the diagnostic yield was anywhere between $59 \%$ and $77 \% \cdot{ }^{[42,43]}$

One study was done by Eberhardt et al., evaluated the procedure in 92 nodules and reported a yield of $67 \%{ }^{[44]}$ The same author and his group compared the use of EBUS only, electromagnetic navigational bronchoscopy (ENB) only, and a combined procedure in 118 patients who eventually had a definitive histological diagnosis by surgical resection. The ENB had a yield of around 59\%. However, the RB-EBUS arm had a better yield of around $69 \%$. The combined procedure proved more accurate and had a better yield of $88 \%{ }^{[45]}$

In a recent large meta-analysis that included 15 trials with 1033 nodules, the overall diagnostic accuracy was $73.9 \%$, the sensitivity for detecting cancer was $71 \%$, but the main complication was pneumothorax, which occurred in 3.1\% of patients. ${ }^{[46]}$ No studies to date have compared CT guided TTNA to ENB, but the diagnostic yield of TTNA remains higher than what we know so far about that of the ENB.

\section{Drawbacks and complications}

One of the main aspects that prevent the wide use of ENB is the prolonged procedure time. The total amount of time involved in the planning phase as well as the procedure itself may exceed several hours. This also contributes to the already high cost of the procedure, a major disadvantage that makes most centers refrain from utilizing it. ${ }^{[47]}$ On the other hand, the yield of the procedure seems to be dependent on the presence of the "bronchus sign," which essentially means the presence of a bronchus leading to the lesion. This was illustrated in a study by Seijo et al., which evaluated the yield of ENB in 51 patients, and reported a $79 \%$ yield in those who had a bronchus sign (30/38) and only $31 \%$ in those who did not $(4 / 13) \cdot{ }^{[48]}$ One of the main factors influencing diagnostic accuracy are nodule size and location. It seems that accuracy improves from $43.7 \%$ for nodules $<2 \mathrm{~cm}$ to around $77 \%$ for those larger than $3 \mathrm{~cm} \cdot{ }^{[43]}$ Upper lobes nodules also seem to have better accessibility. ${ }^{[43]}$

During the procedure, respiratory movements might result in changing lesion location and alter diagnostic accuracy. Furthermore, other than following a preplanned track, one cannot be sure that the lesion has been reached, reason adding RP-EBUS to ENB resulted in some studies in higher yield, owing to the ability of RP-EBUS to provide the needed confirmation. ${ }^{[40,46]}$ Moreover, if the CT images were taken too long in advance of the procedure, inaccurate characterization of the lesion may result. 
Electromagnetic navigational bronchoscopy still carries many of the risks associated with regular bronchoscopy. Moreover, although the risk of pneumothorax is less with the use of ENB as compared to TTNA, yet it remains a serious and possible complication. ${ }^{[40]}$

\section{MEDICAL PLEUROSCOPY}

\section{Description}

Medical pleuroscopy is a procedure usually performed by medical physicians as compared to video-assisted thoracoscopic surgery (VATS) which is done by the surgeon.

It is not known when the first use of pleuroscopy wasintroduced. Earlier mentioning of the term was found in French and German literature, but a description of the procedure was reported in an 1866 paper by Gordon and his urologist colleague Francis Cruise. ${ }^{[49]}$ Swedish physician, Christian Jacobaeus, is much credited with its official first description in $1910,{ }^{[50]}$ when he detailed the procedure in 2 cases and followed a year later with another report of 35 cases.

In modern times, the patient is usually placed in lateral decubitus position, with the abnormal side up. Once a local anesthetic is applied, an incision is made through the chest wall to the pleura, in which, a port is left in place, to be used for insertion of the scope and other instruments. ${ }^{[1,52]}$

The procedure is done under conscious sedation, as opposed to VATS, where the patient is under general anesthesia, and a double lumen endotracheal tube is used for single lung ventilation. In VATS, multiple ports are usually created. ${ }^{[51,52]}$

The procedure typically includes the use of a rigid scope. However, several studies evaluated the use of a flexible scope as well. More recently, semi-rigid scopes, stiff proximally and flexible distally, have been assessed for use since 1998 and are showing promise in providing advantages of both types of scopes. ${ }^{[53,54]}$

Once the procedure is done, a chest tube is usually placed to allow the introduced air to be suctioned and the lung to expand. This usually can be completed in the recovery area, and the patient can be discharged home same day unless the procedure included lung biopsy or pleurodesis.

\section{Indications and yield}

The medical pleuroscope provides advantage over blind pleural biopsy, in sampling the pleura at multiple sites, and providing a good diagnostic tool in cases of undiagnosed pleural effusions, ${ }^{[55]}$ especially when pleural malignancy, primary or secondary, is in question [Figure 3]. This has

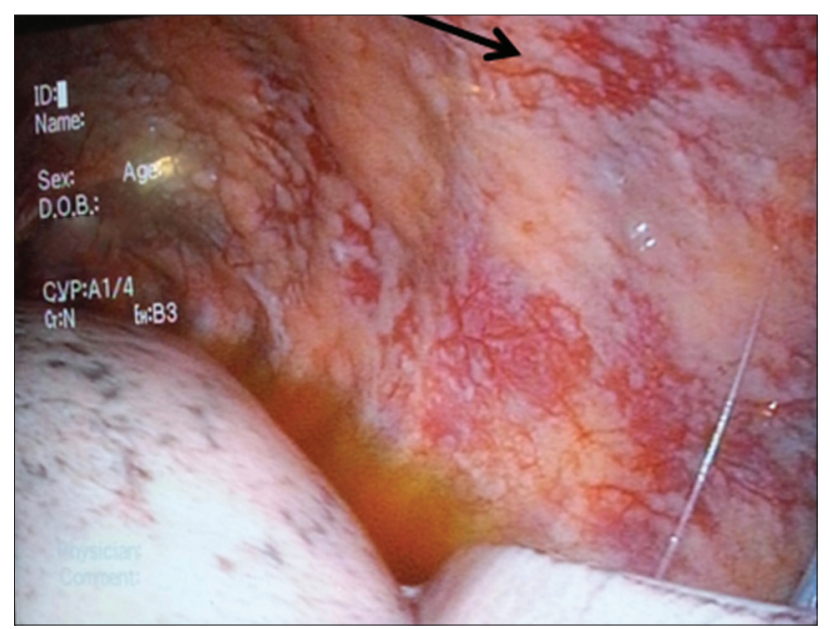

Figure 3: Image obtained during pleuroscopy of the parietal pleura studded with pleural metastasis (black arrow) from adenocarcinoma of the lung

been the primary indication for the use of MP. Unlimited number of biopsy samples can be taken during the procedure, although most authors recommend a minimum of 4-5 samples, from areas of visual abnormalities. If no abnormality is seen, then sampling areas of high lymphatic drainage is also advisable.

Other indications of the procedure include the diagnosis of pleural tuberculosis infections and as a next step, after transbronchial biopsy and bronchoalveolar lavage, in the diagnosis of interstitial lung disease. A pleuroscopy guided lung biopsy can establish the diagnosis and perhaps, can be considered before open lung biopsy or VATS. ${ }^{[6,57]}$

Medical pleursocpy has historically been used for therapeutic purposes as well, which are beyond the scope of this article. However, it is worth to mention that the procedure has mainly been used for two indications; drainage of infected pleural effusions and for pleurodesis. In the case of infected pleural effusions (empyema), the medical pleuroscope can be used for the purpose of breaking adhesions, and aiding in the placement of chest tubes ${ }^{[58]}$ When performing pleurodesis, talc can be instituted by two main methods, talc slurry or talc poudrage. Both types are being studied in large trials to evaluate the efficacy of either.

The diagnostic yield of MP in the case of pleural malignancy varies according to different studies and seems to be anywhere between $70 \%$ and $100 \%$, although most studies report an above $90 \%$ yield. ${ }^{[52,59-61]}$ This is comparable to that of VATS, ${ }^{[52]}$ and higher than that $(60 \%)$ for pleural fluid cytology alone. ${ }^{[62]}$ In the case of tuberculous pleural disease, MP was shown by Diacon et al. to have higher diagnostic yield when compared to blind biopsy or analysis of pleural fluid, with a sensitivity approaching $100 \% .^{[63,64]}$ 
Vansteenkiste et al. obtained lung biopsies in 24 patients with undiagnosed interstitial lung disease using MP. He was able to establish a diagnosis in 18 of them. Although this is not widely used, further studies are needed to confirm the utility of MP in this field. ${ }^{[57]}$

\section{Drawbacks and complications}

The main contraindication to MP is the inability of the lung to collapse, perhaps due to the advanced formation of pleural plaques and fibrotic tissue. Otherwise, the procedure should not be done in patients with undiagnosed cause of hypoxemia, or those who cannot tolerate the lateral decubitus position. Other usual contraindications to any invasive procedure, such as bleeding diathesis, or hemodynamic instability, also apply.

The procedure, although safe, remains an invasive one. One should consider the potential complications of hemorrhage, infection, persistent air leak, and tumor invasion at the incision site. Mortality is very rare, and the complication rate was reported in some studies to be as low as $2.1 \%{ }^{[65,66]}$

\section{Other diagnostic techniques}

Virtual bronchoscopy

Virtual bronchoscopy uses CT scan images to construct a three-dimensional computer generated images of the tracheobronchial tree. A bronchial tract is constructed from CT images and displayed on the virtual image of the bronchial tree. This is similar in concept to the idea of ENB described above, but in this technique, GPS guidance is not used. Instead, the images generated in virtual bronchoscopy are displayed while performing real-time bronchoscopy, and the physician role is to correlate the path between both, in an effort to reach a target point closest to the peripheral lesion, from where, the biopsy instruments are advanced. An imaging modality is needed to confirm placement of biopsy instruments. Hence, virtual bronchoscopy is used in combination with CT guided ultrathin bronchoscopy (a technique that requires high dose radiation), fluoroscopic bronchoscopy, or EBUS with guide sheath. ${ }^{[67]}$

Virtual bronchoscopy allows for the display of areas beyond a stenosis, and the display of extramural structures using the volume rendering method. Hence, virtual bronchoscopy has been used for the evaluation of airway stenosis, tracheal injury, endobronchial malignancy, airway lesions in children, foreign bodies in the airway, and postoperative bronchial complications. ${ }^{[68]}$

The diagnostic yield of virtual bronchoscopy for peripheral lesions varies. There are many small studies evaluating its usefulness, and the reported yield in those studies was anywhere between $44 \%$ and $80 \%$, depending on the imaging modality used with virtual bronchoscopy. ${ }^{[67,69-71]}$

Virtual bronchoscopy seems to improve diagnostic yield for accessing peripheral lesions, when compared to flexible bronchoscopy. However, many disadvantages are present. CT imaging has limitations in visualizing bronchi peripheral to segmental bronchi, demonstrating reduced consistency with actual anatomic findings. Therefore, the use of virtual bronchoscopy has been limited to the central bronchi in many centers. ${ }^{[72]}$ Also, the experience of the physician in correlating images, especially at areas of bifurcating bronchi, seems to play a role in improving diagnostic yield. ${ }^{[72]}$

\section{Autofluorescence bronchoscopy}

Autofluorescence Bronchoscopy was first introduced in the early 1990s. Since then, the various systems using this technique underwent many enhancements, paving the road for it to be moved from the research only area to the day to day clinical use. In most current systems, autofluorescence and regular white light bronchoscopy are built together in one scope, allowing the intermittent use of either.

This technique uses specific substances called fluorophores, which concentrate in the pathologically altered endobronchial mucosa differently than the normal mucosa. This translates into a green color if normal mucosa and magenta or red-brownish color if abnormal, when seen with auto fluorescence imaging as opposed to white light bronchoscopy. ${ }^{[73]}$

The procedure is used for the detection of endobronchial lesions, mainly suspected of having a precancerous nature. In one large meta-analysis, the pooled sensitivity and specificity of Autofluorescence Bronchoscopy was 0.90 and 0.56 respectively. ${ }^{[74]}$ The specificity varied among other studies due to factors that include the prevalence of cancer and the program used. Better specificity, however, is noted when it is used in the follow-up investigation of surgical margins after resection surgery. Also, many authors advocate the use of this technique to detect synchronous lung cancers. ${ }^{[75,76]}$

One major problem with this technique is that only small percentage of dysplastic lesions progresses to carcinoma in situ (CIS) or invasive cancer. ${ }^{[77]}$ The unknown natural history of endobronchial dysplastic lesions makes this technique unlikely to be routinely used. As a result, the American College of Chest Physicians recommended its use when sputum cytology is positive, or when the patient is considered for endobronchial therapy to treat CIS, or when a known history of central airway severe dysplasia or CIS exists. ${ }^{[78]}$ 


\section{Narrow band imaging}

This is a technology that enables an extensive and detailed examination of the submucosal microcapillary bed. In concept, it uses light wavelengths that are preferentially absorbed by hemoglobin permitting a better identification of microvascular structures. Since dysplastic lesions have increased angiogenesis, the technique can detect early dysplasia. ${ }^{[79]}$ The patterns of submucosal blood vessels seen with this technique are normal pattern, abnormal patterns that are not pathological and known as background noise, or pathologically abnormal patterns, which are the ones targeted. Shibuya et al., studied in 2003, the usefulness of narrow band imaging (NBI) in detecting what is known as "angiogenic squamous dysplasia" a known precancerous lesion. The study included heavy smokers, of whom, many had sputum cytology positive for malignant cells. It found a great correlation between the abnormal pathological patterns detected by NBI and the true "angiogenic squamous dysplasia" confirmed on pathology ${ }^{[80]}$ Herth et al. evaluated the diagnostic yields of NBI individually and in combination with regular white light bronchoscopy and autofluorescence bronchoscopy. The sensitivity of NBI was similar to that of autofluorescence bronchoscopy and higher than white light bronchoscopy. However, the specificity was found to be higher than autofluorescence bronchoscopy. ${ }^{[81]}$ The technology seems to be useful overall in detecting early dysplastic lesions, but as with autofluorescence bronchoscopy, it is uncertain to what extent is can be applied, due to the vague natural history of endobronchial dysplastic lesions.

\section{CONCLUSION}

Diagnostic pulmonary procedures have evolved to a great level in the last few decades. Regular flexible bronchoscopy is rarely used these days to sample mediastinal and hilar lymph nodes, due to the better diagnostic accuracy with EBUS. For peripheral lung lesions, the pulmonologist now has the option to use RP EBUS or ENB to obtain samples. The yield for either procedure is comparable to that of CT guided biopsy with fewer complications. The advances in diagnostic pulmonary interventions are resulting in earlier and more specific diagnosis of pulmonary lesions [Table 2],

Table 2: The diagnostic yield of the different
\begin{tabular}{lc} 
bronchoscopic procedures in the diagnosis of lung cancer \\
\hline Diagnostic procedure & Yield for lung cancer (\%) \\
\hline Flexible bronchoscopy & 41.4 (peripheral lesions) $^{[10]}$ \\
RP-EBUS & 73 (peripheral lesions) $^{[26]}$ \\
CP-EBUS & 79 (mediastinal nodes) $^{[36]}$ \\
ENB & 73.9 (peripheral lesions) $^{[46]}$ \\
Virtual bronchoscopy & $44-80$ (peripheral lesions) $^{[63,65,67]}$ \\
\hline RP-EBUS=Radial probe endobronchial ultrasound, CP-EBUS=Curvilinear probe \\
endobronchial ultrasound, ENB=Electromagnetic navigational bronchoscopy
\end{tabular}

which may translate into more effective treatment and better outcome

\section{REFERENCES}

1. Haas AR, Vachani A, Sterman DH. Advances in diagnostic bronchoscopy. Am J Respir Crit Care Med 2010;182:589-97.

2. Ikeda S, Yanai N, Ishikawa S. Flexible bronchofiberscope. Keio J Med 1968; 17:1-16.

3. Becker HD. Bronchoscopy: The past, the present, and the future. Clin Chest Med 2010;31:1-18.

4. Casal RF, Ost DE, Eapen GA. Flexible bronchoscopy. Clin Chest Med 2013;34:341-52.

5. Mason RJ, Broaddus VC, Murray JF, Nadel JA. In: Murray and Nadel editors. Textbook of Respiratory Medicine. $4^{\text {th }}$ ed. Philadelphia, Pa, USA: Elsevier-Saunders; 2005.

6. Funahashi A, Lohaus GH, Politis J, Hranicka LJ. Role of fibreoptic bronchoscopy in the diagnosis of mycobacterial diseases. Thorax 1983;38:267-70.

7. Baughman RP. Use of bronchoscopy in the diagnosis of infection in the immunocompromised host. Thorax 1994;49:3-7.

8. Jain P, Sandur S, Meli Y, Arroliga AC, Stoller JK, Mehta AC. Role of flexible bronchoscopy in immunocompromised patients with lung infiltrates. Chest 2004;125:712-22.

9. Ensminger SA, Prakash UB. Is bronchoscopic lung biopsy helpful in the management of patients with diffuse lung disease? Eur Respir J 2006;28:1081-4.

10. Rittirak W, Sompradeekul S. Diagnostic yield of fluoroscopy-guided transbronchial lung biopsy in non-endobronchial lung lesion. J Med Assoc Thai 2007;90 Suppl 2:68-73.

11. Schenk DA, Bower JH, Bryan CL, Currie RB, Spence TH, Duncan CA, et al. Transbronchial needle aspiration staging of bronchogenic carcinoma. Am Rev Respir Dis 1986;134:146-8.

12. Ernst A, Eberhardt R, Wahidi M, Becker HD, Herth FJ. Effect of routine clopidogrel use on bleeding complications after transbronchial biopsy in humans. Chest 2006;129:734-7.

13. Hürter T, Hanrath P. Endobronchial sonography: Feasibility and preliminary results. Thorax 1992;47:565-7.

14. Kurimoto N, Murayama M, Yoshioka S, Nishisaka T, Inai K, Dohi K. Assessment of usefulness of endobronchial ultrasonography in determination of depth of tracheobronchial tumor invasion. Chest 1999;115:1500-6.

15. Tanaka F, Muro K, Yamasaki S, Watanabe G, Shimada Y, Imamura M, et al. Evaluation of tracheo-bronchial wall invasion using transbronchial ultrasonography (TBUS). Eur J Cardiothorac Surg 2000;17:570-4.

16. Baba M, Sekine Y, Suzuki M, Yoshida S, Shibuya K, lizasa T, et al. Correlation between endobronchial ultrasonography (EBUS) images and histologic findings in normal and tumor-invaded bronchial wall. Lung Cancer 2002;35:65-71.

17. Herth F, Ernst A, Schulz M, Becker H. Endobronchial ultrasound reliably differentiates between airway infiltration and compression by tumor. Chest 2003;123:458-62.

18. Herth FJ, Becker HD, Ernst A. Ultrasound-guided transbronchial needle aspiration: An experience in 242 patients. Chest 2003;123:604-7.

19. Herth F, Becker HD, Ernst A. Conventional vs endobronchial ultrasound-guided transbronchial needle aspiration: A randomized trial. Chest 2004;125:322-5.

20. Herth FJ, Lunn W, Eberhardt R, Becker HD, Ernst A. Transbronchial versus transesophageal ultrasound-guided aspiration of enlarged mediastinal lymph nodes. Am J Respir Crit Care Med 2005;171:1164-7.

21. Kurimoto N, Murayama M, Yoshioka S, Nishisaka T. Analysis of the internal structure of peripheral pulmonary lesions using endobronchial ultrasonography. Chest 2002;122:1887-94. 
22. Chao TY, Lie CH, Chung YH, Wang JL, Wang YH, Lin MC. Differentiating peripheral pulmonary lesions based on images of endobronchial ultrasonography. Chest 2006;130:1191-7.

23. Herth FJ, Eberhardt R, Becker HD, Ernst A. Endobronchial ultrasound-guided transbronchial lung biopsy in fluoroscopically invisible solitary pulmonary nodules: A prospective trial. Chest 2006;129:147-50.

24. Kurimoto N, Miyazawa T, Okimasa S, Maeda A, Oiwa H, Miyazu Y, et al. Endobronchial ultrasonography using a guide sheath increases the ability to diagnose peripheral pulmonary lesions endoscopically. Chest 2004;126:959-65.

25. Steinfort DP, Khor YH, Manser RL, Irving LB. Radial probe endobronchial ultrasound for the diagnosis of peripheral lung cancer: Systematic review and meta-analysis. Eur Respir J 2011;37:902-10.

26. Wang Memoli JS, Nietert PJ, Silvestri GA. Meta-analysis of guided bronchoscopy for the evaluation of the pulmonary nodule. Chest 2012;142:385-93.

27. Czarnecka K, Yasufuku K. Interventional pulmonology: Focus on pulmonary diagnostics. Respirology 2013;18:47-60.

28. Yasufuku K, Chiyo M, Sekine Y, Chhajed PN, Shibuya K, lizasa T, et al. Real-time endobronchial ultrasound-guided transbronchial needle aspiration of mediastinal and hilar lymph nodes. Chest 2004;126:122-8.

29. Gu P, Zhao YZ, Jiang LY, Zhang W, Xin Y, Han BH. Endobronchial ultrasound-guided transbronchial needle aspiration for staging of lung cancer: A systematic review and meta-analysis. Eur J Cancer 2009;45:1389-96.

30. Dong X, Qiu X, Liu Q, Jia J. Endobronchial ultrasound-guided transbronchial needle aspiration in the mediastinal staging of non-small cell lung cancer: A meta-analysis. Ann Thorac Surg 2013;96:1502-7.

31. Varela-Lema L, Fernández-Villar A, Ruano-Ravina A. Effectiveness and safety of endobronchial ultrasound-transbronchial needle aspiration: A systematic review. Eur Respir J 2009;33:1156-64.

32. Yasufuku K, Pierre A, Darling G, de Perrot M, Waddell T, Johnston M, et al. A prospective controlled trial of endobronchial ultrasound-guided transbronchial needle aspiration compared with mediastinoscopy for mediastinal lymph node staging of lung cancer. J Thorac Cardiovasc Surg 2011;142:1393-400.e1.

33. Andrade RS, Podgaetz E, Rueth NM, Majumder K, Hall E, Saric C, et al. Endobronchial ultrasonography versus mediastinoscopy: A single-institution cost analysis and waste comparison. Ann Thorac Surg 2014;98:1003-7.

34. Fritscher-Ravens A, Davidson BL, Hauber HP, Bohuslavizki KH, Bobrowski C, Lund C, et al. Endoscopic ultrasound, positron emission tomography, and computerized tomography for lung cancer. Am J Respir Crit Care Med 2003;168:1293-7.

35. Tremblay A, Stather DR, Maceachern P, Khalil M, Field SK. A randomized controlled trial of standard vs endobronchial ultrasonography-guided transbronchial needle aspiration in patients with suspected sarcoidosis. Chest 2009;136:340-6.

36. Agarwal R, Srinivasan A, Aggarwal AN, Gupta D. Efficacy and safety of convex probe EBUS-TBNA in sarcoidosis: A systematic review and meta-analysis. Respir Med 2012;106:883-92.

37. Asano F, Aoe M, Ohsaki Y, Okada Y, Sasada S, Sato S, et al. Complications associated with endobronchial ultrasound-guided transbronchial needle aspiration: A nationwide survey by the Japan Society for Respiratory Endoscopy. Respir Res 2013;14:50.

38. Haas AR, Vachani A, Sterman DH. Advances in diagnostic bronchoscopy. Am J Respir Crit Care Med 2010;182:589-97.

39. Schwarz Y, Mehta AC, Ernst A, Herth F, Engel A, Besser D, et al. Electromagnetic navigation during flexible bronchoscopy. Respiration 2003;70:516-22.

40. Leong S, Ju H, Marshall H, Bowman R, Yang I, Ree AM, et al. Electromagnetic navigation bronchoscopy: A descriptive analysis. J Thorac Dis 2012;4:173-85.
41. Schwarz Y, Greif J, Becker HD, Ernst A, Mehta A. Real-time electromagnetic navigation bronchoscopy to peripheral lung lesions using overlaid CT images: The first human study. Chest 2006;129:988-94.

42. Lamprecht B, Porsch P, Pirich C, Studnicka M. Electromagnetic navigation bronchoscopy in combination with PET-CT and rapid on-site cytopathologic examination for diagnosis of peripheral lung lesions. Lung 2009;187:55-9.

43. Makris D, Scherpereel A, Leroy S, Bouchindhomme B, Faivre JB, Remy J, et al. Electromagnetic navigation diagnostic bronchoscopy for small peripheral lung lesions. Eur Respir J 2007;29:1187-92.

44. Eberhardt R, Anantham D, Herth F, Feller-Kopman D, Ernst A. Electromagnetic navigation diagnostic bronchoscopy in peripheral lung lesions. Chest 2007;131:1800-5.

45. Eberhardt R, Anantham D, Ernst A, Feller-Kopman D, Herth F. Multimodality bronchoscopic diagnosis of peripheral lung lesions: A randomized controlled trial. Am J Respir Crit Care Med 2007;176:36-41.

46. Gex G, Pralong JA, Combescure C, Seijo L, Rochat T, Soccal PM. Diagnostic yield and safety of electromagnetic navigation bronchoscopy for lung nodules: A systematic review and meta-analysis. Respiration 2014;87:165-76.

47. Dale CR, Madtes DK, Fan VS, Gorden JA, Veenstra DL. Navigational bronchoscopy with biopsy versus computed tomography-guided biopsy for the diagnosis of a solitary pulmonary nodule: A cost-consequences analysis. J Bronchology Interv Pulmonol 2012;19:294-303.

48. Seijo LM, de Torres JP, Lozano MD, Bastarrika G, Alcaide AB, Lacunza MM, et al. Diagnostic yield of electromagnetic navigation bronchoscopy is highly dependent on the presence of a Bronchus sign on CT imaging: Results from a prospective study. Chest 2010;138:1316-21.

49. Gordon S. Clinical reports of rare cases, occurring in the Whit worth and Hardwicke Hospitals: Most extensive pleuritic effusion rapidly becoming purulent, paracentesis, introduction of a drainage tube, recovery, examination of interior of pleura by the endoscope. Dubl Q J Med Sci 1866;41:83-90.

50. Jacobaeus HC. The Possibilities for Performing Cystoscopy in Examinations of Serous Cavities (Uber die Moglichkeit, die Zystoskopie bei Untersuchungen seroser Hohlungenanzuwenden). Munch Med Wochenschr 1910;40:2090-2.

51. Lee P, Colt HG. Pleuroscopy in 2013. Clin Chest Med 2013;34:81-91.

52. Bhatnagar R, Maskell NA. Medical pleuroscopy. Clin Chest Med 2013;34:487-500.

53. Munavvar M, Khan MA, Edwards J, Waqaruddin Z, Mills J. The autoclavable semirigid thoracoscope: The way forward in pleural disease? Eur Respir J 2007;29:571-4.

54. Rozman A, Camlek L, Kern I, Malovrh MM. Semirigid thoracoscopy: An effective method for diagnosing pleural malignancies. Radiol Oncol 2014;48:67-71.

55. Wilsher ML, Veale AG. Medical thoracoscopy in the diagnosis of unexplained pleural effusion. Respirology 1998;3:77-80.

56. Rodgers BM, Moazam F, Talbert JL. Thoracoscopy. Early diagnosis of interstitial pneumonitis in the immunologically suppressed child. Chest 1979;75:126-30.

57. Vansteenkiste J, Verbeken E, Thomeer M, Van Haecke P, Eeckhout AV, Demedts M. Medical thoracoscopic lung biopsy in interstitial lung disease: A prospective study of biopsy quality. Eur Respir J 1999;14:585-90.

58. Kern L, Robert J, Brutsche M. Management of parapneumonic effusion and empyema: Medical thoracoscopy and surgical approach. Respiration 2011;82:193-6.

59. Weissberg D, Kaufmann M. Diagnostic and therapeutic pleuroscopy. Experience with 127 patients. Chest 1980;78:732-5.

60. Sakuraba M, Masuda K, Hebisawa A, Sagara Y, Komatsu H. Diagnostic value of thoracoscopic pleural biopsy for pleurisy under local anaesthesia. ANZ J Surg 2006;76:722-4. 
61. Davies HE, Nicholson JE, Rahman NM, Wilkinson EM, Davies RJ, Lee YC. Outcome of patients with nonspecific pleuritis/fibrosis on thoracoscopic pleural biopsies. Eur J Cardiothorac Surg 2010;38:472-7.

62. Maskell NA, Butland RJ, Pleural Diseases Group, Standards of Care Committee, British Thoracic Society. BTS guidelines for the investigation of a unilateral pleural effusion in adults. Thorax 2003;58 Suppl 2:ii8-17.

63. Diacon AH, Van de Wal BW, Wyser C, Smedema JP, Bezuidenhout J, Bolliger CT, et al. Diagnostic tools in tuberculous pleurisy: A direct comparative study. Eur Respir J 2003;22:589-91.

64. Loddenkemper R, Mai J, Scheffler N, Brandt HJ. Prospective individual comparison of blind needle biopsy and of thoracoscopy in the diagnosis and differential diagnosis of tuberculous pleurisy. Scand J Respir Dis Suppl 1978;102:196-8.

65. Brims FJ, Arif M, Chauhan AJ. Outcomes and complications following medical thoracoscopy. Clin Respir J 2012;6:144-9.

66. Metintas M, Ak G, Yildirim H, Danacioglu S, Dundar E, Metintas S. The safety of medical thoracoscopy in a group at high risk for complications. J Bronchology Interv Pulmonol 2013;20:224-31.

67. Asano F. Virtual bronchoscopic navigation. Clin Chest Med 2010;31:75-85

68. Amorico MG, Drago A, Vetruccio E, Bollino F, Pizzuti G, Gallo E. Tracheobronchial stenosis: Role of virtual endoscopy in diagnosis and follow-up after therapy. Radiol Med 2006;111:1064-77.

69. Shinagawa N, Yamazaki K, Onodera Y, Asahina H, Kikuchi E, Asano F, et al. Factors related to diagnostic sensitivity using an ultrathin bronchoscope under CT guidance. Chest 2007;131:549-53.

70. Tachihara M, Ishida T, Kanazawa K, Sugawara A, Watanabe K, Uekita K, et al. A virtual bronchoscopic navigation system under X-ray fluoroscopy for transbronchial diagnosis of small peripheral pulmonary lesions. Lung Cancer 2007;57:322-7.

71. Asahina H, Yamazaki K, Onodera Y, Kikuchi E, Shinagawa N, Asano F, et al. Transbronchial biopsy using endobronchial ultrasonography with a guide sheath and virtual bronchoscopic navigation. Chest 2005;128:1761-5.

72. Lacasse Y, Martel S, Hébert A, Carrier G, Raby B. Accuracy of virtual bronchoscopy to detect endobronchial lesions. Ann Thorac Surg 2004;77:1774-80.
73. Zaric B, Perin B, Becker HD, Herth FF, Eberhardt R, Djuric M, et al. Autofluorescence imaging videobronchoscopy in the detection of lung cancer: From research tool to everyday procedure. Expert Rev Med Devices 2011;8:167-72.

74. Chen W, Gao X, Tian Q, Chen L. A comparison of autofluorescence bronchoscopy and white light bronchoscopy in detection of lung cancer and preneoplastic lesions: A meta-analysis. Lung Cancer 2011;73:183-8.

75. van Rens MT, Schramel FM, Elbers JR, Lammers JW. The clinical value of lung imaging fluorescence endoscopy for detecting synchronous lung cancer. Lung Cancer 2001;32:13-8.

76. Piérard P, Faber J, Hutsebaut J, Martin B, Plat G, Sculier JP, et al. Synchronous lesions detected by autofluorescence bronchoscopy in patients with high-grade preinvasive lesions and occult invasive squamous cell carcinoma of the proximal airways. Lung Cancer 2004;46:341-7.

77. Breuer RH, Pasic A, Smit EF, van Vliet E, Vonk Noordegraaf A, Risse EJ, et al. The natural course of preneoplastic lesions in bronchial epithelium. Clin Cancer Res 2005;11:537-43.

78. Kennedy TC, McWilliams A, Edell E, Sutedja T, Downie G, Yung R, et al. Bronchial intraepithelial neoplasia/early central airways lung cancer: ACCP evidence-based clinical practice guidelines ( $2^{\text {nd }}$ edition). Chest 2007;132:221S-33.

79. Shibuya K, Nakajima T, Fujiwara T, Chiyo M, Hoshino H, Moriya Y, et al. Narrow band imaging with high-resolution bronchovideoscopy: A new approach for visualizing angiogenesis in squamous cell carcinoma of the lung. Lung Cancer 2010;69:194-202.

80. Shibuya K, Hoshino H, Chiyo M, lyoda A, Yoshida S, Sekine Y, et al. High magnification bronchovideoscopy combined with narrow band imaging could detect capillary loops of angiogenic squamous dysplasia in heavy smokers at high risk for lung cancer. Thorax 2003;58:989-95.

81. Herth FJ, Eberhardt R, Anantham D, Gompelmann D, Zakaria MW, Ernst A. Narrow-band imaging bronchoscopy increases the specificity of bronchoscopic early lung cancer detection. J Thorac Oncol 2009;4:1060-5.

Cite this article as: Al-Zubaidi N, Soubani AO. Advances in diagnostic interventional pulmonology. Avicenna J Med 2015;5:57-66

Source of Support: Nil, Conflict of Interest: None declared.

\section{"Quick Response Code" link for full text articles}

The journal issue has a unique new feature for reaching to the journal's website without typing a single letter. Each article on its first page has a "Quick Response Code". Using any mobile or other hand-held device with camera and GPRS/other internet source, one can reach to the full text of that particular article on the journal's website. Start a QR-code reading software (see list of free applications from http://tinyurl.com/ yzlh2tc) and point the camera to the QR-code printed in the journal. It will automatically take you to the HTML full text of that article. One can also use a desktop or laptop with web camera for similar functionality. See http://tinyurl.com/2bw7fn3 or http://tinyurl.com/3ysr3me for the free applications. 